\title{
L’hélice de la vie
}

Axel Kahn

> La découverte de la double hélice d'ADN, il y a 50 ans, constitue l'événement fondateur de la biologie moléculaire. C'est aussi à cette occasion que se forgea la réputation de deux des figures les plus fascinantes de la biologie. Cependant, Jim Watson et Francis Crick ne furent pas les seuls acteurs de cet extraordinaire épisode de l'histoire des sciences dont certaines circonstances restent aujourd'hui assez singulières. <

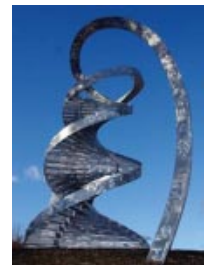

la nucléine est appelé « acide nucléique », puis ADN, distingué alors de

I'ARN. Mais c'est là une substance abondante, de constitution chimique simple et qui semble par conséquent indigne de jouer un rôle dans le phénomène le plus noble de la biologie, la transmission des caractères héréditaires. Les protéines apparaissent être plus complexes, et on les sait dotées de nombreuses propriétés biologiques. Les diastases, ou enzymes, sont, en particulier, des protéines. On considère donc ces dernières comme seules capables d'être les constituants des gènes. En 1944, le grand physicien théoricien Erwin Schrödinger écrit un ouvrage intitulé Qu'est-ce que la vie? La vision de Schrödinger selon laquelle les gènes sont des composants clés des cellules vivantes, et par conséquent que les progrès en biologie nécessitent de les connaître mieux, connaîtra un retentissement considérable. C'est en cette même année 1944 qu'une prodigieuse expérience est réalisée par l'Américain Oswald Th. Avery et ses collaborateurs, Colin MacLeod et Maclyn McCarty: le caractère génétique de virulence de souches de bactéries, des pneumocoques, peut être transféré non par l'intermédiaire des protéines, mais par celui de I'ADN [1]. Peu après, McCarty et Avery confirment leurs résultats en démontrant que le pouvoir transformant de l'extrait bactérien est inactivé par la DNAse. Quoiqu'il s'agisse là, en réalité, d'un résultat comparable par son importance à la détermination de la structure en double hélice de l'ADN, et peut-être même «The pivotal discovery of the 20th century biology » comme le dira, 50 ans après, le biochimiste Joshua Lederberg, 0.T. Avery n'obtiendra néanmoins jamais le Prix Nobel. De nombreux biologistes doutent en effet encore que les gènes soient constitués 


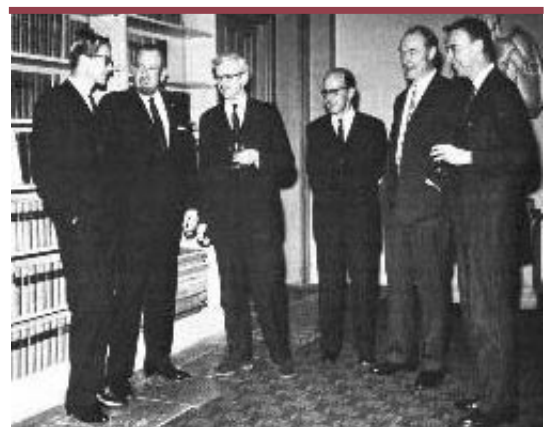

d'ADN et non de protéines. Il faudra attendre 1952 et les travaux des Américains Alfred Hershey et Martha Chase, puis 1953 avec la description de la structure de I'ADN, pour achever de convaincre la communauté scientifique de la nature chimique réelle des gènes. Hershey et Chase montrent en effet que le matériel infectant d'un bactériophage, celui qui pénètre dans la bactérie, est surtout constitué d'ADN, et non de protéines [2]. Or, le phage est alors considéré comme un agent biologique constitué de gènes presque purs capables d'infecter des bactéries, de se répliquer et de s'exprimer. C'est la raison pour laquelle le « groupe du phage », dont James Watson est un jeune produit, va jouer un rôle de pionnier dans l'émergence de la biologie moléculaire, avec Salvador Luria et Max Delbrück installés à

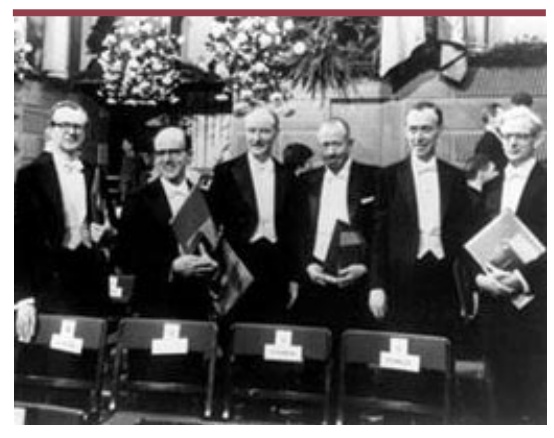
Caltech, Californie, depuis 1947, et aussi André Lwoff et ses collaborateurs à I'Institut Pasteur de Paris. Avery meurt en 1957, trop peu de temps après l'éclatante confirmation du rôle de I'ADN pour que la modification des mentalités ne se manifeste jusqu'au sein du Comité Nobel.

\section{Le théâtre et les acteurs d'une découverte}

James Watson, quant à lui, n'avait pas attendu cette confirmation pour être convaincu par Schrödinger que les gènes constituaient la clé de la vie, et par Avery qu'ils étaient constitués chimiquement d'ADN. C'est dans le but de mieux comprendre le fonctionnement des gènes que Luria proposa au jeune Watson, âgé de 22 ans, d'acquérir les bases nécessaires pour en entreprendre l'étude chimique. C'est ainsi que, après un passage à Copenhague, Watson se retrouva en 1951 au laboratoire Cavendish de Cambridge, alors dirigé par Sir Lawrence Bragg, créateur, avec son père, de la prestigieuse école britannique d'étude cristallographique de molécules biologiques. Au Danemark, le jeune Watson avait été passionné par le plus récent succès du grand chimiste Linus Pauling (Pasadena, Californie, USA), dans l'établissement de la structure des protéines. L'élément de base en était constitué d'une hélice, structure apparaissant attractive pour rendre compte de nombreux objets biologiques. Cet intérêt de
Watson pour la structure hélicoïdale s'accentua lorsqu'il eut l'occasion d'assister à une conférence du physicien cristallographe anglais Maurice Wilkins. Ce dernier montra en effet un diagramme sommaire de diffraction aux rayons $X$ d'un cristal d'ADN témoignant d'une régularité que pouvait expliquer une structure en hélice. Au Cavendish, Watson s'associa rapidement, dans le groupe dirigé par Max Perutz, à un stagiaire doctoral déjà âgé, physicien curieux et brillant théoricien des structures cristallines, Francis Crick. C'est principalement l'histoire de la collaboration entre ce jeune américain de 23 ans et cet étudiant anglais un peu attardé, esprit original de 35 ans, que racontera dans le détail James Watson, dans son ouvrage La double hélice [3]. Les protagonistes de l'aventure de Crick et de Watson sont avant tout leurs collègues de Cambridge, qui leur apportent les éléments techniques et scientifiques dont ils ont besoin, leur permettant souvent de rectifier leurs erreurs; les responsables d'équipes dans le laboratoire de Lawrence Bragg, Max Perutz et son collègue John Kendrew, qui perpétuent la tradition anglaise de cristallographie en tentant de résoudre la structure de I'hémoglobine et de la myoglobine et obtiendront le Prix Nobel de Chimie en 1962, l'année même où le prix Nobel de Physiologie et Médecine récompense Watson, Crick et Wilkins; Linus Pauling, dont on sait qu'il s'intéresse luiaussi à la structure de l'ADN et dont le propre fils, Peter, est un collègue de Crick et Watson à Cambridge; et, enfin, l'équipe de biophysique du King's College de Londres avec Maurice Wilkins et sa collaboratrice Rosalind Franklin. Au départ, Crick et Watson, qui n'ont eux-mêmes jamais réalisé d'expériences sur l'ADN et sur ses composants, et qui ne le feront pas plus entre 1951 et 1953, ne disposent pratiquement d'aucune donnée pour atteindre leur but, c'està-dire résoudre la structure de l'ADN. Pourtant, ils veulent utiliser la méthode si brillamment illustrée par Linus Pauling, à savoir parvenir au résultat grâce à la constitution d'un modèle moléculaire réalisé par l'assemblage précis, en trois dimensions, des différents atomes et molécules constituant l'ADN. Encore fallait-il avoir accès, pour parvenir à la constitution d'un modèle crédible, à des données objectives qui manquent au départ totalement à nos deux héros. Les seuls renseignements objectifs sur la structure de l'ADN étudiée par diffraction aux rayons $X$ proviennent du laboratoire de Wilkins à Londres. Or, ce dernier ne voit pas avec sympathie ces jeunes novices venir le défier dans un domaine qu'il tente patiemment de défricher depuis des années. De plus, la situation au King's College est terriblement tendue. Le patron du laboratoire, John Randal, a confié à une nouvelle venue, la jeune Rosalind Franklin, âgée de 30 ans, l'essentiel du travail sur l'étude cristallographique de I'ADN. Les relations entre Rosalind Franklin et Wilkins sont rapidement épouvantables, empê- 
chant toute communication. Rosalind Franklin est une brillante cristallographe, expérimentatrice hors pair, une femme moderne et libre, exigeant le respect. Elle ne supporte pas l'atmosphère du laboratoire qu'elle rejoint en 1951, marqué par des liens hiérarchiques très stricts et un solide mépris pour les femmes, surtout quand elles sont d'origine juive. Les scientifiques des deux sexes ne prennent le plus souvent pas leur repas dans la même salle et tout indique que les chercheurs-femmes ne sont guère considérées que comme des collaboratrices subalternes. Cela, Rosalind Franklin ne le supporte pas. Ses relations seront également très mauvaises avec le jeune James Watson, qui l'évitera d'ailleurs systématiquement pour ne s'adresser qu'à Wilkins. La personnalité de Rosalind Franklin, ses relations avec Wilkins et le duo du Cavendish, sa contribution effective à l'élucidation de la structure de l'ADN, ont été l'objet de nombreuses analyses qui tendent en général à atténuer l'image peu amène qu'en donne Watson dans son ouvrage paru en 1968 [3-5]. Ce dernier, dans l'épilogue de l'édition de 1968 [3], rendait ainsi un « pieux hommage » à cette jeune biophysicienne au destin tragique. La nouvelle édition de La double hélice, parue en 2002, aux États-Unis et en 2003 en France [6], atténue quelque peu le caractère abrupt des jugements portés sur elle. Contrairement à tous les autres acteurs de l'aventure scientifique contée ici, Rosalind Franklin (photo ci-contre) ne connaîtra pas les honneurs; sa carrière s'arrêtera en effet en 1958, à l'âge de 37 ans, stoppée net par l'évolution d'un cancer de l'ovaire. On peut penser que, sinon, le Jury Nobel aurait eu bien des hésitations pour couronner soit Wilkins, soit Rosalind. En un hommage tardif, le King's College de Londres, où Rosalind Franklin s'était sentie si peu à l'aise, donnera son nom à l'un de ses importants bâtiments de recherche.

\section{Une année de doute}

Lorsque Crick et Watson montrent un premier modèle de leur cru à Rosalind Franklin et à Maurice Wilkins à Noël 1951, ces derniers n'ont aucun mal à en démontrer le caractère totalement erroné. On sait que I'ADN doit être constitué de deux types d'éléments: un squelette formé de sucre et de phosphate; et des bases, au nombre de quatre, adénine, thymine, guanine et cytosine $(A, T, G, C)$. Dans le premier modèle, Watson et Crick commencent à proposer que le squelette soit au centre, lié par des ions magnésium qui interagissent avec les groupes phosphates. Les bases se retrouvent alors à l'extérieur. Cette hypothèse n'est cohérente ni avec les données cristallographiques, ni même avec la vraisemblance stéréochimique. Nos deux héros sont alors priés par leurs supérieurs de cesser d'empiéter sur le domaine des cristallographes du King's College, et de les laisser poursuivre sereinement, à leur rythme, leur patient labeur de professionnels expérimentés. Francis Crick retourne à sa thèse sur l'étude de la structure de l'hémoglobine, sous la direction de Max Perutz, alors que James Watson papillonne brillamment, effleurant différents sujets à la lisière entre la génétique et la biologie structurale. Il est intéressant à ce stade de bien marquer la différence entre les démarches des différents protagonistes de cet épisode essentiel de l'histoire des sciences qui se joue. Pour Maurice Wilkins et Rosalind Franklin, I'ADN n'est guère qu'une structure moléculaire complexe à élucider grâce à l'application rigoureuse des méthodes cristallographiques qui ont établi la réputation de l'école britannique, notamment à la suite des travaux des Braggs, père et fils. La démarche des équipes du King's College est parallèle à celle de Max Perutz sur l'hémoglobine et de John Kendrew sur la myoglobine. Francis Crick, lui-même physicien reconverti à la biologie, est un produit de cette école de biophysiciens structuralistes, mais aussi un esprit impertinent et pétillant, d'une infinie curiosité, toujours à l'affût d'un concept nouveau et excitant. Pour le jeune Watson, la vision et la démarche sont tout autres. Sa quête est en effet celle du Saint Graal de la biologie, la structure et la nature du gène, molécule clé de la vie. Le jeune américain espère que ce sera là le moyen de comprendre la grande énigme du programme génétique: il se perpétue identique à lui-même lorsque les cellules se divisent, mais peut être sujet à des altérations spontanées ou provoquées par des interventions physiques et chimiques, facteurs de mutations. Watson et Crick ne doutent pas un seul instant que la célébrité attende ceux qui parviendront à un tel succès. Le Prix Nobel est même probablement à la clé.

\section{L'assaut final}

Après un an de doutes, c'est la conjonction de trois types d'événements et circonstances qui va donner le coup d'envoi du sprint final pour l'élucidation de la structure de I'ADN. Tout d'abord, Linus Pauling informe son fils Peter que, après les protéines, il s'est tourné vers la structure de I'ADN, et l'a élucidée. Cependant, un examen rapide de la solution proposée par Pauling convainc immédiatement Watson, Crick et tous leurs collègues qu'elle

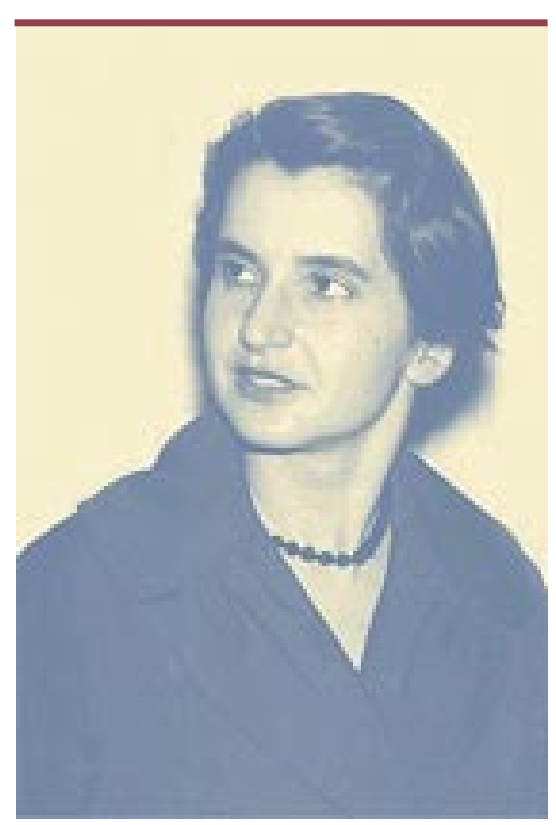


est erronée. En effet, Pauling imagine une triple hélice avec les squelettes de sucre et de phosphate à l'intérieur et les bases à l'extérieur. Le tout est assemblé par des ponts hydrogènes, en réalité inimaginables entre des molécules ionisées de résidus phosphate légèrement acides. La gaffe est tellement grossière qu'elle sera sans doute bien vite signalée à Pauling qui, probablement, mettra tout en œuvre pour la corriger et aboutir sans délai à une structure exacte. La situation est donc chaude. Par ailleurs, Rosalind Franklin est parvenue à obtenir des images de diffraction aux rayons $X$ de préparations d'ADN de conformations homogènes. Elle a reconnu que les difficultés antérieurement rencontrées dans l'interprétation des diagrammes de diffraction étaient liées à l'existence de l'ADN sous deux formes selon le degré d'hydratation. Dans les échantillons antérieurs, la forme $A$, déshydratée et quelque peu contrainte, prédominait. Mais, Rosy, ainsi que l'appelle Watson dans son ouvrage $[3,6]$, parvient à obtenir des préparations homogènes d'ADN hydraté dans sa configuration B. Les diagrammes de diffraction sont alors d'une qualité remarquable, et d'interprétation beaucoup plus évidente. Ils confirment une déduction antérieure de Rosalind Franklin selon laquelle le squelette de la molécule se trouve à l'extérieur et les bases à l'intérieur. De plus, il est relativement aisé de déduire de ces images le diamètre du brin

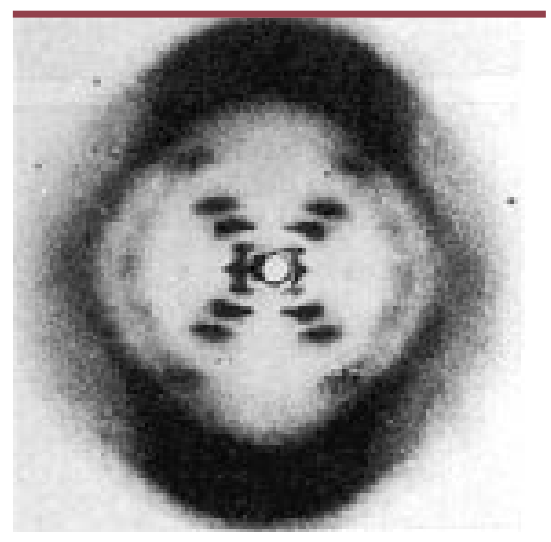
d'ADN, sa périodicité et le nombre probable de chaînes qu'il contient. Ces données fondamentales parviennent à Watson de manière détournée, par l'intermédiaire de Maurice Wilkins, d'une part, et également de Max Perutz auquel les résultats ont été communiqués de façon confidentielle par John Randall, le supérieur hiérarchique des équipes du

King's College. Cette information est contenue dans les documents transmis aux chercheurs ayant la charge de procéder à une évaluation scientifique des laboratoires de biophysique de Londres. Watson se met au travail, aidé et conseillé par Crick. II rencontre encore des obstacles, liés à ses lacunes en chimie. Les erreurs seront surmontées grâce à deux circonstances supplémentaires. Tout d'abord, le biochimiste américain Edwin Chargaff, d'origine autrichienne, vient de démontrer que, dans les molécules d'ADN, on observe que l'abondance des adénines est toujours identique à celle des thymines, et l'abondance des guanines identique à celle des cytosines. Par ailleurs, Cambridge est sans doute alors l'un des endroits au monde les plus propices à la promotion de la créativité scientifique. S'y côtoient en effet des équipes pluridisciplinaires de chercheurs de haut niveau, passionnés par la science et avides d'échanger des idées, concepts et informations. C'est ce qui permet à Watson de corriger un modèle intermédiaire auquel il parvient. Le squelette est bien à l'extérieur, formant une double hélice, mais les barreaux en reliant les deux brins sont constitués de contacts entre des bases identiques: $A$ avec $A, G$ avec $G$, etc. Un collègue signale que cette solution est impossible du fait de la conformation réelle des bases. Émerge alors, construite par assemblage de pièces modélisant les constituants, à la manière d'un Légo, la célébrissime double hélice affinée par le théoricien Crick et recevant l'onction des spécialistes consultés en chimie et en cristallographie, en particulier Maurice Wilkins et Rosalind Franklin. Les deux brins sont disposés en sens opposé et forment une double hélice dont le pas est de 34 Angström; ils sont reliés par des contacts spécifiques, en l'occurrence des ponts hydrogènes entre $A$ et $T$, $G$ et $C$. Ainsi, les brins sont précisément complémentaires I'un de l'autre. Après leur séparation, il suffit que chacun d'entre eux serve de matrice à la synthèse d'un nouveau brin complémentaire pour que soient reconstituées les deux doubles hélices qui se trouveront dans les deux cellulesfilles issues de la division d'une cellule-mère.

La solution est simple et belle. L'esthétique ne se réfère pas simplement ici à l'élégance intellectuelle permettant de parvenir au résultat, mais aussi à l'harmonie de la forme. Pour beaucoup de scientifiques, et en particulier pour les théoriciens, une telle harmonie est l'un des critères amenant à considérer qu'un modèle peut être vrai. La nature ne procède en effet pas par caprice, mais plutôt par exécution selon des lois dont il revient aux scientifiques de percer les secrets. Ainsi, la régularité et l'harmonie sont-elles de fréquentes conséquences de ce processus auquel n'échappe pas même la sélection naturelle darwinienne. Ici, les modifications de la double hélice responsables des mutations sont certes aléatoires, mais la nature élimine ce qui ne convient pas, et ne conserve que ce qui s'adapte correctement à un milieu donné. Raisonnablement assurés de leur modèle, encore purement théorique et ne reposant que sur des données expérimentales acquises par une autre, Watson et Crick écrivent rapidement un très bref manuscrit, illustré d'un schéma unique et sommaire réalisé par Odile Crick, la femme de Francis. II est entendu que cet article [7] sera soumis à Nature parallèlement à ceux rapportant les travaux de Maurice Wilkins et de ses collaborateurs, Alec Stockes et Herbert Wilson [8], et de Rosalind Franklin et de son étudiant, Raymond Gosling [9]. La date de soumission est le 2 avril 1953, celle de publication le 25... un délai d'une brièveté record. Crick fit ajouter au manuscrit la phrase aujourd'hui fameuse « Il n'a pas échappé à notre 
attention que l'appariement spécifique des bases que nous avons postulé suggère immédiatement un possible mécanisme du recopiage du matériel génétique ». En effet, la structure de la double hélice est un événement fondateur de la biologie et de la génétique moderne. Compte tenu de l'importance de cette discipline dans la société moderne et à venir, il s'agit là aussi d'un événement historique de premier ordre.

\section{En guise de conclusion: triomphe et éthique}

Quoique l'importance de la découverte mît quelques années encore avant d'être définitivement reconnue par toute la communauté des biologistes, la double hélice est aujourd'hui une icône d'une célébrité sans pareille, largement amplifiée par le succès de l'ouvrage de Watson [3, 6]. Les conditions de la mise au point du modèle et de la publication de 1953 marquent aussi une époque et une situation qui apparaissent aujourd'hui singulières. Le lapidaire article de Watson et Crick dans le numéro du 25 avril de Nature ne repose en effet sur aucune donnée expérimentale personnelle, mais sur des résultats dérobés à une collègue, ce qu'elle ignorera d'ailleurs jusqu'à sa mort. Des documents confidentiels destinés à une évaluation scientifique sont détournés, et frauduleusement communiqués à de jeunes concurrents des équipes évaluées. II n'existe au début aucune vérification expérimentale du modèle (il faudra attendre pour cela la démonstration par Matthew Meselson et Franklin Stahl de la nature semi-conservatrice de la réplication de l'ADN [10]). Le manuscrit est immédiatement accepté et est publié en trois semaines. Les standards admis de la déontologie, de l'éthique, et même de la rigueur scientifique, sont malmenés. Et pourtant, sans aucun doute, cet article de moins d'une page, fruit de circonstances étonnantes, et même choquantes, est l'un des textes les plus importants de la science, voire du monde d'aujourd'hui. En fêter le cinquantenaire s'impose donc comme une évidence. $\diamond$

\section{RÉFÉRENCES}

1. Avery OT, Macleod CM, McCarty M. Studies on the chemical nature on the substance inducing transformation of pneumoccocal types. Induction of transformation by a deoxyribonucleic acid fraction isolated from Pneumococcus type III. J Exp Med 1944; 79: 137-58.

2. Hershey AD, Chase M.
Independent functions of viral protein and nucleic acid in growth of bacteriophage. J Gen Physiol 1952; 36: 39-56.

3. Watson J. The double helix. A personal account of the discovery of the structure of DNA. New York: Athenum, $1968: 226$ p.

4. Sayre A. Rosalind Franklin and DNA. New York: W.W. Norton and Co, $1975: 240$ p.

5. Maddox B. Rosalind

\section{SUMMARY}

The helix of life

The discovery of DNA's double helix 50 years ago was the founding event of molecular biology. It was also the moment that forged the reputation of two of biology's most compelling figures, no doubt in perpetuity. However, Jim Watson and Francis Crick were not the only players of this outstanding fest whose certain circumstances remain today rather singular. $\diamond$

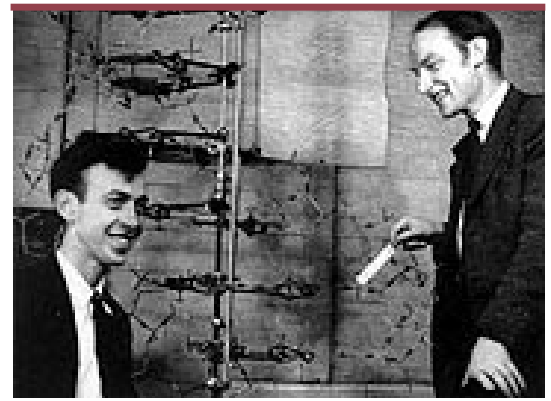

\section{LÉGENDES DES ILLUSTRATIONS}

Page 492. - Les lauréats avant la remise des Prix Nobel. Maurice Wilkins, John Steinbeck, John Kendrew, Max Perutz, Francis Crick et James Watson (Stockholm, décembre 1962).

- Cérémonie de remise des Prix Nobel 1962, Maurice Wilkins, Max Perutz, Francis Crick, John Steinbeck, James Watson, John Kendrew (Stockholm, décembre 1962).

Page 493. Rosalind Franklin (1920-1958).

Page 494. Diagramme de diffraction aux rayons $X$ de préparations d'ADN réalisé par Rosalind Franklin.

Page 495. James Watson, Francis Crick et la célébrissime double hélice.
Franklin. The dark lady of DNA. London: Harper Collins, $2002: 380 \mathrm{p}$.

6. Watson J. La double hélice. Paris: Robert Laffont, 2003

7. Watson J, Crick FH. A structure for deoxyribose nucleic acid. Nature 1953; 171: 737-8.

8. Wilkins MHF, Stokes AR, Wilson HR. Molecular structure of deoxypentose nucleic acids. Nature 1953; 171: 738-40.
9. Franklin RE, Gosling RG. Molecular configuration in sodium thymonucleate. Nature 1953; 171: 740-1.

10. Meselson M, Stahl FW. The replication of DNA in Escherichia coli. Proc Natl Acad Sci USA 1958; 44: 671-82.
TIRÉS À PART

A. Kahn 